\title{
Evidências de Validade do Questionário de Relacionamento Central - CRQ 6.0 em Universitários
}

\author{
Ademir dos Santos" \& Elisa Medici Pizão Yoshida \\ Pontifícia Universidade Católica de Campinas, Campinas, Brasil
}

\begin{abstract}
RESUMO
Teve-se como objetivo avaliar a consistência interna e estrutura fatorial do Questionário de Relacionamento Central - CRQ 6.0, desenvolvido para avaliar o padrão de relacionamento amoroso/conflituoso, segundo três componentes: Desejo (D), Resposta do Outro (RO) e Resposta do Eu (RE). Amostra de 509 universitários (73,28\% mulheres), de três estados (PR, SP e SC). A coleta de dados com o CRQ 6.0 foi coletiva, em sala de aula. Subamostras divididas por sexo, universidade pública e privada e estados da federação não diferiram quanto aos escores médios dos componentes. Obteve-se boa consistência interna de cada componente e para o total. A análise fatorial (varimax) reproduziu dois fatores: RE e D. Um terceiro, Controle do Relacionamento, representou domínio e controle do parceiro.
\end{abstract}

Palavras-chave: padrão de relacionamento; autorrelato; avaliação psicológica.

\begin{abstract}
Evidences of Validity of the Central Relationship Questionnaire-CRQ 6.0 with college students This study aimed to evaluate the internal consistency and factorial structure of the Central Relationship Questionnaire - CRQ 6.0, developed to measure the pattern of romantic/conflictual relationship according to three components: Wish (W), Response from Other (RO) and Response of Self (RS). The sample was composed of 509 college students (undergraduates) (73.28\% women) from three country states (PR, SP and SC). Data was collectively collected. The subscales divided by sex gender, public and private universities and country states did not differ regarding the mean scores of the components. It was obtained a good internal consistency from each component. The factorial analysis (varimax) yielded two factors: RS and W. A third component, Control on the Relationship, represented dominance and control of the partner.
\end{abstract}

Keywords: pattern of relationship; self-report; psychological evaluation.

A pesquisa objetivou contribuir para o processo de validação da versão em português do Questionário de Relacionamento Central - CRQ (Barber, Foltz \& Weinryb, 1998). O CRQ é um instrumento do tipo autorrelato, desenvolvido para avaliar padrões de relacionamento disfuncionais, conforme o conceito proposto por Lester Luborsky e conhecido como Core Conflcitual Relationship Theme - CCRT (Luborsky, 1984; Luborsky \& Crits-Christoph, 1998).

O CCRT é um procedimento clínico de avaliação do conflito relacional central do paciente, segundo três dimensões: Desejo (D) - que são necessidades ou expectativas sobre algo do outro ou do grupo com quem o sujeito se relaciona; Resposta do Outro (RO) - esperar ou ter expectativas sobre as respostas do outro fantasiando ou antecipando estas respostas; Resposta do Eu (RE) - desenvolver fantasias antecipadas sobre suas próprias respostas, às respostas do outro, em forma de emoções, comportamentos ou sintomas (Luborsky \& Crits-Christoph, 1998). A avaliação do CCRT está baseada na identificação da presença destas dimensões na narrativa do paciente, ao longo do processo psicoterapêutico. Apesar de ser um dos métodos mais empregados para a avaliação de mudança no padrão relacional conflituoso em pesquisas

\footnotetext{
Endereço para correspondência: Ademir dos Santos - ademprof@gmail.com.
} 
de processo e de resultados de psicoterapias (CritsChristoph, Barber, Grenyer \& Diguer, 2010; Lhullier, 1998) o CCRT apresenta algumas desvantagens que foram apontadas por Barber et al. (1998), para justificar o desenvolvimento de uma versão de autorrelato intitulada, Questionário de Relacionamento Central CRQ. Dentre elas estão: a necessidade de transcrever literalmente as sessões de psicoterapia e, no momento da avaliação, submetê-las à apreciação de juízes independentes e experientes. Esta última etapa, por sua vez, demanda longos períodos de tempo, além de nem sempre ser fácil obter um bom nível de acordo entre os juízes.

A versão original do CRQ (Barber et al., 1998) teve como objetivo avaliar os padrões de relacionamento conflituoso, com o parceiro amoroso. Sendo que se entende por parceiro amoroso, a pessoa com quem se esteja relacionando de forma romântica e/ou sexual, no momento, ou nos últimos três anos. Mais recentemente, uma versão modificada do CRQ foi desenvolvida, destinada a avaliar o padrão de conflito relacional do sujeito também com outras pessoas relevantes pai, mãe, irmãos, melhor amigo (McCarthy, Gibbons \& Barber, 2008). No entanto, como a versão em português do CRQ baseou-se na original, neste artigo serão focalizados apenas os estudos que empregaram o instrumento designado para avaliar o padrão conflituoso com o parceiro amoroso.

Uma pesquisa, com a versão original do CRQ (Barber et al., 1998), apontou boas qualidades psicométricas do instrumento. Alfas de Cronbach de amostras de estudantes universitários $(n=315)$ e de pessoas da comunidade com diferentes diagnósticos (ansiedade, pânico, agorafobia, pânico com agorafobia, fobias socias, fobia simples, transtorno obsessivo-compulsivo, estresse pós-traumático, ansiedade generalizada), segundo o DSM-III-R ( $\mathrm{n}=96)$, variaram entre $0,75 \mathrm{e}$ 0,95 para Desejos, 0,82 e 0,95 para Resposta do Outro e 0,71 e 0,94 para Resposta do Eu. Foram ainda estimadas a precisão de teste e reteste (após um ano) e medidas de validade convergente e divergente, com o emprego de diversos instrumentos de avaliação da personalidade e de sintomas psicopatológicos. Concluiu-se que o CRQ permitia identificar, de forma confiável, os padrões de relacionamento interpessoal mais característico num relacionamento amoroso e que as evidências de validade ratificavam as expectativas teóricas quanto ao fato de divergirem ou convergirem com medidas de sintomas e problemas interpessoais.
Posteriormente, o CRQ foi adaptado para o sueco (Weinryb, Barber, Foltz, Göransson \& Gustavsson, 2000), e uma pesquisa, nos mesmos moldes da anterior (Barber et al., 1998), foi realizada. A amostra foi composta por um grupo de estudantes universitários $(60 \mathrm{M}$ e $31 \mathrm{H})$, um grupo de pacientes $(15 \mathrm{M}$ e $15 \mathrm{H})$ suecos e um grupo de estudantes americanos (49 $\mathrm{M} \mathrm{e}$ $49 \mathrm{H})$. Os resultados apontaram que a versão sueca do CRQ também possuía aceitável consistência interna, com alfas entre 0,84 e 0,95 para amostra de universitários e 0,65 e 0,96 para a amostra clínica. Comparou-se ainda os resultados dos estudantes americanos e suecos, utilizando um modelo misto de análise de variância ajustado para grupos. Neste caso, o objetivo foi verificar eventuais diferenças culturais. Resumidamente, obteve-se na subescala Desejo, que os suecos demonstram mais desejos de ser sexi e ser reconhecidos do que os americanos, enquanto as suecas expressaram muito mais conflitos com o parceiro em relação ao componente Desejo, do que as americanas. Em RO, as mulheres americanas avaliaram seus pares românticos como altamente significativos, o mesmo não ocorrendo com as suecas. Quanto à RE, os resultados sugeriram que os estudantes americanos apresentaram maiores escores para a subescala ser independente, ajudar o outro e evitar conflitos, do que os suecos. Apesar destas diferenças, os autores concluíram que a versão sueca do CRQ permite avaliar de forma satisfatória o padrão conflituoso com parceiros românticos e que seus resultados são comparáveis aos da versão em inglês.

O CRQ foi traduzido e adaptado para o português do Brasil por Rocha (2007), a partir da versão 6.0 do instrumento (descrito no método), motivo pelo qual tem sido identificado no Brasil como CRQ 6.0, em pesquisas que buscaram determinar algumas de suas qualidades psicométricas (Massei \& Yoshida, 2009; Risso \& Yoshida, 2010; Silva \& Yoshida, 2009). Nelas, foi avaliada a consistência interna e obtidas evidências de validade convergente e discriminante, junto a mulheres vítimas de violência (Silva \&Yoshida, 2009), portadores de HIV/AIDS (Massei \& Yoshida, 2009) e pacientes com diagnóstico de Hepatitie C (Risso \& Yoshida, 2010).

A pesquisa de Silva e Yoshida (2009), com mulheres vítimas de violência, baseou-se em amostra de 54 participantes divididas em Grupo $1(\mathrm{n}=32)$, composto por mulheres assistidas em uma instituição de acolhimento de mulheres vítimas de violência, e Grupo 2 
$(n=22)$, integrado por mulheres que se encontravam internadas em maternidade de hospital geral. No Grupo 1 os escores médios e respectivos desvios padrão de cada componente foram: $\mathrm{D}=4,55(0,76) ; \mathrm{RO}=$ $4,31(0,74)$ e $\mathrm{RE}=4,20(0,50)$ e no Grupo $2: \mathrm{D}=$ $4,93(0,63) ; \mathrm{RO}=4,19(0,87)$; $\mathrm{RE} 4,47(0,70)$, portanto valores muito próximos para os dois grupos. A consistência interna do CRQ 6.0 para a amostra total e para cada grupo foi avaliada por meio de coeficientes alfa de Cronbach. Na amostra total obteve-se 0,82 para Desejo, 0,69 para Resposta do Outro e 0,71 para Resposta do Eu. No Grupo 1 os valores de alfa foram, respectivamente, $0,80,0,76$ e 0,71 e no Grupo 2, 0,83, 0,68 e 0,59 .

A validade convergente foi avaliada estimando-se a associação do CRQ 6.0 com a Escala de Avaliação de Sintomas (EAS-40) (Laloni, 2001). Os resultados confirmaram parcialmente a expectativa de associação entre a severidade dos sintomas psicopatológicos e o conflito no relacionamento amoroso, uma vez que foram observadas correlações significantes $(\mathrm{p}<0,05)$ entre os componentes RO e RE do CRQ 6.0 e o escore total da EAS-40 do Grupo Caso. Além disso, neste grupo foram encontradas correlações significantes entre estes componentes do CRQ 6.0 e duas dimensões da EAS-40 (psicoticismo e obsessividadecompulsividade). No Grupo Controle, obteve-se correlação significante apenas entre o componente D e a dimensão obsessividade-compulsividade da EAS-40 (Silva \& Yoshida, 2009). O estudo de validade discriminante foi realizado comparando-se o CRQ 6.0 das participantes do Grupo Caso e Grupo Controle. Não foram obtidas diferenças significantes, sugerindo que mulheres vítimas de violência não apresentam maior intensidade de conflito nos relacionamentos amorosos quando comparadas a mulheres parturientes. As autoras advertem, todavia, para a necessidade de novas pesquisas, com amostras mais representativas e randomizadas (Silva \& Yoshida, 2009).

Estudo semelhante foi realizado por Massei e Yoshida (2009) com amostra composta por 90 participantes $(45 \mathrm{M} \mathrm{e} 45 \mathrm{H})$, divididos em um grupo de pacientes com diagnóstico de HIV/AIDS $(20 \mathrm{M}$ e $30 \mathrm{H})$ e um grupo de pacientes de diversas enfermarias de um hospital universitário $(25 \mathrm{M}$ e $15 \mathrm{H})$. No grupo caso (pacientes) os escores médios e respectivos desvios padrão foram os seguintes: $\mathrm{D}=2,2(0,58) ; \mathrm{RO}=2,88$ $(1,04) ; \mathrm{RE}=2,71(0,75)$. E no grupo controle (universitários): $\mathrm{D}=2,4(0,75) ; \mathrm{RO}=3,15(1) ; \mathrm{RE}=3,09(0,74)$.
Como no estudo de Silva e Yoshida (2009) não foram observadas diferenças significantes $(p<0,05)$ entre os escores médios dos grupos. Os alfas variaram entre 0,80 e 0,87 nos três componentes (D, RO e RE). Em relação à validade convergente os resultados apontaram correlações significantes $(\mathrm{p}<0,05)$ entre os três componentes do CRQ 6.0 e o escore total da EAS-40 (Laloni, 2001) e também para as dimensões psicoticismo e obsessividade-compulsividade da EAS-40, para a amostra total e o Grupo de portadores de HIVAIDS (com exceção de RO). Na amostra total também houve associações significantes entre RE e as dimensões somatização e ansiedade da EAS-40 sugerindo, portanto, a existência de associação entre o conflito relacional com o parceiro amoroso e a severidade de sintomas psicopatológicos. A comparação do Grupo de portadores de HIV/AIDS e de pacientes de enfermaria, quanto aos resultados do CRQ 6.0, mostrou que eles diferem apenas em relação ao RE, com escores significantemente mais altos entre os participantes com HIV/AIDS, e que as mulheres desse grupo apresentam escores mais elevados que os homens. Concluiu-se que o componente RE permite discriminar portadores de HIV/AIDS de não portadores e que o nível de conflito entre as mulheres é ainda mais intenso, se comparado ao dos homens (Massei \& Yoshida, 2009).

Risso e Yoshida (2010) avaliaram a consistência interna, validade convergente e validade discriminante do CRQ 6.00 junto à amostra de pacientes portadores de Hepatite C crônica. A amostra, de 101 (49M e 52H) participantes, ficou dividida em Grupo 1, composto por pacientes ambulatoriais com Hepatite C crônica (24M e $37 \mathrm{H})$ e Grupo 2, composto por acompanhantes de pacientes de outros ambulatórios do mesmo hospital universitário $(25 \mathrm{M}$ e $15 \mathrm{H})$. Como no estudo de Massei e Yoshida (2009), os escores médios ficaram abaixo dos de Silva e Yoshida (2009) e também não se observou diferenças significantes entre eles. Foram as seguintes as médias e desvios padrão de cada grupo: Grupo 1: $\mathrm{D}=2,43(0,68) ; \mathrm{RO}=3,47(0,99) ; \mathrm{RE}=3,13$ $(0,82)$. No Grupo 2: $\mathrm{D}=2,22(0,58)$; $\mathrm{RO}=2,88(1,04)$; $\mathrm{RE}=2,71(0,75)$. Os alfas de Cronbach variaram entre 0,80 e 0,87 , indicando, portanto, boa consistência interna dos três componentes do CRQ 6.0. Também foram encontradas associações significantes $(p<0,05)$ entre eles e a EAS-40 (Laloni, 2001), corroborando a expectativa teórica de associação entre a severidade de sintomas psicopatológicos e conflitos interpessoais em pacientes com Hepatite C crônica. E, no estudo de 
validade discriminante (Tabela 2), os participantes do Grupo 1 tiveram escores superiores $(p<0,05)$ aos do Grupo 2 nos componentes RO e RE do CRQ 6.0. Além disso, os participantes do Grupo 1 que tomavam medicação, apresentaram escores superiores em RE, e as mulheres, quando comparadas aos homens, em D e $\mathrm{RE}$, indicando que estes substratos apresentam maiores níveis de conflito relacional, dentre os portadores de Hepatite $\mathrm{C}$ crônica. Os autores concluíram que o CRQ 6.0 pode ser considerado um instrumento adequado para a avaliação de conflitos relacionais nesta população, embora alertem para as limitações da pesquisa (Risso \& Yoshida, 2010).

Assim como os estudos internacionais com as versões do CRQ que medem o padrão de conflito central no relacionamento amoroso (Barber et al., 1998, Weinryb et al., 2000), as pesquisas realizadas com a versão brasileira (Massei \&Yoshida; Risso \& Yoshida, 2010; Silva \& Yoshida, 2009) não incluíram a análise fatorial uma vez que as evidências de validade de construto foram consideradas suficientes, pelos autores. Os autores utilizaram a validação convergente e discriminante para a validade de construto verificando assim, que o teste estabelece correlações significativas com variáveis com as quais deve se relacionar e não a estabelecendo com aquelas com as quais não tem correlação teórica (Anastasi \& Urbina, 2000). Os alfas de Cronbach contribuíram com a decisão, visto que os mesmos demonstraram haver significativa consistência interna do construto.

Nas diversas pesquisas efetuadas com o CRQ (Barber et al. 1998; Weinryb et al. 2000, Silva \& Yoshida, 2009; Massei \&Yoshida; Risso \& Yoshida, 2010) nenhum tipo de análise fatorial foi utilizada. Especialmente, em se tratando de uma versão adaptada, a avaliação da estrutura fatorial é necessária devido, sobretudo, a fatores de ordem semântica. O presente estudo teve, portanto, como objetivo preencher esta lacuna. A versão do CRQ utilizada foi traduzida e adaptada para o português do Brasil por Rocha (2007), a partir da versão 6.0 da escala original.

Além disso, como os estudos internacionais com o CRQ basearam-se em amostras de universitários, considerou-se relevante avaliar as propriedades psicométricas da versão em português também para esse estrato da população brasileira. Estabeleceram-se então como objetivos: avaliar a consistência interna do CRQ
6.0 e realizar um estudo de validade de construto por meio da análise de sua estrutura fatorial.

O CRQ, versão original americana (Barber et al., 1998), compôs-se pelos já citados três fatores: D- que são necessidades ou expectativas sobre algo do outro ou do grupo com quem o sujeito se relaciona; RO esperar ou ter expectativas sobre as respostas do outro fantasiando ou antecipando estas respostas; RE-desenvolver fantasias antecipadas sobre suas próprias respostas, às respostas do outro, em forma de emoções, comportamentos ou sintomas (Luborsky \& Crits-Christoph, 1998). A formulação destes três fatores baseou-se no CCRT (Luborsky \& Crits-Christoph, 1998).

\section{MÉTODO}

\section{Participantes}

Estudantes, de três estados brasileiros (SP, PR e SC), compuseram a amostra de 509 participantes. Para a definição do tamanho mínimo da amostra foi utilizado o critério da "razão itens/sujeitos" segundo o qual a amostra deve ter entre 5 e 10 sujeitos por item do instrumento. O CRQ é composto por 101 itens, assim, a amostra correspondeu ao critério de 5 participantes para cada item. Além disto, este critério satisfaz o requisito exigido para a realização de Análises Fatoriais, que é de se contar ao menos com 200 sujeitos (Pasquali, 1999, 2001).

Quanto ao perfil sociodemográfico, houve predomínio de mulheres (73,29\%); com idades entre 17 e 18 anos $(69,35 \%)$; cursando os dois primeiros anos de graduação $(67,35 \%)$. Os cursos de Pedagogia $(n=223)$ e Administração $(n=160)$ concentraram a maioria dos participantes $(75,24 \%)$. Predominaram estudantes de universidades se pública $(85 \%)$. Em relação à distribuição por estados, 53,04\% eram de universidades do Paraná, 42,63\% de São Paulo e 4,33\% de Santa Catarina.

\section{Instrumento de Medida}

Foi utilizada a versão em português do Questionário de Relacionamento Central - CRQ 6.0 (Rocha, 2007). Trata-se de instrumento de autorrelato desenvolvido para avaliar o padrão de conflito amoroso segundo três dimensões: Desejo (D), Resposta do Outro (RO), Resposta do Eu (RE). Antes de responder aos itens relativos às dimensões do instrumento é 
pedido ao respondente que classifique seu parceiro amoroso quanto à importância dele em sua vida. Para tal ele responde a seis questões: 1) O quanto essa pessoa é ou foi próxima de você? 2) O quanto é ou foi íntimo seu relacionamento com essa pessoa? 3) Quanto essa pessoa representa ou representava uma figura de autoridade para você? 4) Quanto essa pessoa é ou foi importante para você? 5) Quanto esse relacionamento é ou foi agradável nos melhores momentos? 6) Quanto esse relacionamento é ou foi difícil nos piores momentos? As repostas são dadas segundo uma escala Likert de sete pontos, sendo que 1 demonstra dificuldades no relacionamento, como distanciamento falta de intimidade, autoritarismo, grau de importância e seu comportamento nos melhores e piores momentos de sua vida; enquanto que 7 demonstra facilidades no relacionamento. O objetivo é o de utilizar estas questões para uma eventual avaliação clínica dos conflitos relacionais e ajudar o respondente a refletir sobre seus sentimentos em relação ao parceiro amoroso. É explicado a ele que o parceiro amoroso é aquele com quem esteja se relacionando no momento, ou esteve nos últimos três anos, e que este relacionamento tenha aspectos românticos e/ou sexuais (Barber et al., 1998). Em seguida, solicita-se que sejam respondidas as 101 afirmações que compõem o CRQ, propriamente dito. São 40 itens para o componente D; 23 para RO e 38 para RE. Os itens são também pontuados segundo uma escala Likert de 7 pontos, sendo que 1 representa situações ruins no relacionamento e 7 as melhores situações.

\section{Procedimento}

Obteve-se primeiramente autorização dos dirigentes das universidades envolvidas. Foram feitas aplicações coletivas nas salas de aula dos alunos. Em algumas universidades o próprio professor fez a aplicação e remeteu os dados, via correio aos pesquisadores, em outras o primeiro autor fez a coleta de dados. Instruções sobre o procedimento de aplicação dos instrumentos foram fornecidas a todos os aplicadores para garantir sua padronização. Primeiramente, era dada uma explicação dos objetivos da pesquisa e se sublinhava o caráter voluntário da participação, em seguida à assinatura do Termo de Consentimento Livre e Esclarecido, os questionários eram distribuídos e os alunos recebiam então uma orientação quanto ao preenchimento e a importância de seguirem as orientações constantes no mesmo. Cada participante respondeu ao
CRQ 6.0, individualmente. O projeto de pesquisa foi aprovado pelo Comitê de Ética da instituição dos pesquisadores (Processo 407/09).

\section{RESULTADOS}

\section{Médias e Desvio Padrão}

A Tabela 1 traz os valores médios e respectivos desvios padrão de cada componente do CRQ 6.0 (D, RO e RE) para as variáveis: sexo e tipo de universidade - se pública ou privada. Há também as probabilidades associadas às diferenças, estimadas por meio de testes $t$ de Student. As diferenças entre as médias de cada componente de acordo como o estado e o tipo de curso foram estimadas por meio da ANOVA (Tabela 2). Os resultados demonstram não haver diferenças significantes entre as médias dos três componentes do $\mathrm{CRQ}$, em relação às variáveis estudadas.

\section{Consistência Interna}

Para a aferição da consistência interna do CRQ foram estimados os coeficientes alfa de Cronbach. Os resultados indicaram consistência interna satisfatória $(\alpha>0,70)$ para a escala do CRQ 6.0 total $(0,92)$ e em cada componente: $\mathrm{D}=0,86, \mathrm{RO}=0,82$ e $\mathrm{RE}=0,81$.

\section{Validade de Construto}

O estudo de validade de construto foi realizado por meio da análise da estrutura fatorial do CRQ 6.0. Para verificar a adequação dos dados à análise fatorial foi, inicialmente, estimada a Medida de Adequação da Amostra (MSA) do teste Kaiser-Meyer-Olkin (KMO) (Johnson \& Wichern, 1988). Obteve-se o valor 0,87 , que confirma a fatorabilidade da versão em português da escala. A seguir, foi realizada Análise Fatorial por meio do método dos componentes principais. A primeira análise, com os 101 itens, foi feita por solução livre. Conforme o esperado, esta não se mostrou uma solução interessante, dada a quantidade de componentes gerados. Esta tentativa criou uma estrutura com vinte e seis componentes iniciais representando $64,56 \%$ da variância total da escala. Nesta análise, observou-se que os três primeiros componentes juntos representavam $27,82 \%$ da variância, sendo que o fator 1 explicava $15,80 \%$, o fator $2,7,03 \%$ e o fator $3,4,45 \%$ da variância total. A representação gráfica pode ser verificada no scree-plot (Figura 1), onde se destacam entre três e quatro primeiros fatores. Procedeu-se então a 
análise fatorial com rotação varimax para três e quatro fatores. A solução de três fatores resultou em fatores mais claramente interpretáveis e compatíveis com o teoricamente esperado.

Por ser o objetivo principal de o estudo buscar evidências para a validade do instrumento decidiu-se por uma análise fatorial exploratória. Assim, entendeu-se que a rotação varimax com extração de componentes principais seria suficiente para estes objetivos, e de fato ela apresentou os dados de forma clara possibilitando uma análise adequada.

O CRQ foi desenvolvido para mensurar conflitos de relacionamento (Barber et al. 1998) e em sua composição apresenta 3 fatores interdependentes $-\mathrm{D}, \mathrm{RO}$, RE - (Luborsky\& Crits-Christoph, 1998) entendeu-se que uma verificação da estrutura fatorial por meio da análise exploratória aprofundaria as informações.

Tabela 1

Média, Desvio Padrão e Teste t para as variáveis sexo e tipo de universidade

\begin{tabular}{|c|c|c|c|c|c|c|}
\hline Componente & Sexo & $\mathbf{N}$ & Média & Desvio Padrão & $\mathbf{t}$ & $p$ \\
\hline \multirow{2}{*}{ D } & $M$ & 130 & 4,80 & 0,54 & \multirow{2}{*}{0,014} & \multirow{2}{*}{0,99} \\
\hline & $\mathrm{F}$ & 364 & 4,80 & 0,46 & & \\
\hline \multirow{2}{*}{ RO } & M & 133 & 3,98 & 0,52 & \multirow{2}{*}{1,24} & \multirow{2}{*}{0,22} \\
\hline & $\mathrm{F}$ & 352 & 3,89 & 0,49 & & \\
\hline \multirow{2}{*}{$\mathrm{Eu}$} & M & 132 & 4,31 & 0,55 & \multirow{2}{*}{1,02} & \multirow{2}{*}{0,31} \\
\hline & $F$ & 362 & 4,26 & 0,41 & & \\
\hline Componente & Universidade & & & & & \\
\hline \multirow{2}{*}{ D } & Pública & 263 & 4,76 & 0,46 & \multirow{2}{*}{1,86} & \multirow{2}{*}{0,06} \\
\hline & Privada & 231 & 4,85 & 0,51 & & \\
\hline \multirow{2}{*}{ RO } & Pública & 257 & 3,90 & 0,46 & \multirow{2}{*}{0,27} & \multirow{2}{*}{0,8} \\
\hline & Privada & 228 & 3,91 & 0,54 & & \\
\hline \multirow{2}{*}{ RE } & Pública & 262 & 4,24 & 0,41 & \multirow{2}{*}{1,58} & \multirow{2}{*}{0,12} \\
\hline & Privada & 232 & 4,31 & 0,50 & & \\
\hline
\end{tabular}

Tabela 2

Resultados da ANOVA de cada componente do CRQ entre os estados: $S P, P R, S C$

\begin{tabular}{ccccccc}
\hline Componente & Fonte & $\begin{array}{c}\text { Soma dos } \\
\text { quadrados }\end{array}$ & GI & $\begin{array}{c}\text { Média dos } \\
\text { quadrados }\end{array}$ & F & p \\
\hline \multirow{3}{*}{ Desejo } & Entre os grupos & 1,000 & 2 & 2,119 & 0,014 & 0,121 \\
& No grupo & 115,861 & 491 & 0,236 & & \\
& Total & 116,861 & 493 & & & \\
\multirow{2}{*}{ Outro } & Entre os grupos & 0,045 & 2 & 0,023 & 0,089 & 0,915 \\
& No grupo & 122,423 & 482 & 0,254 & & \\
& Total & 122,468 & 484 & & & \\
& Entre os grupos & 0,608 & 2 & 0,384 & 1,432 & 0,240 \\
& No grupo & 104,202 & 491 & 0,212 & & \\
\hline
\end{tabular}




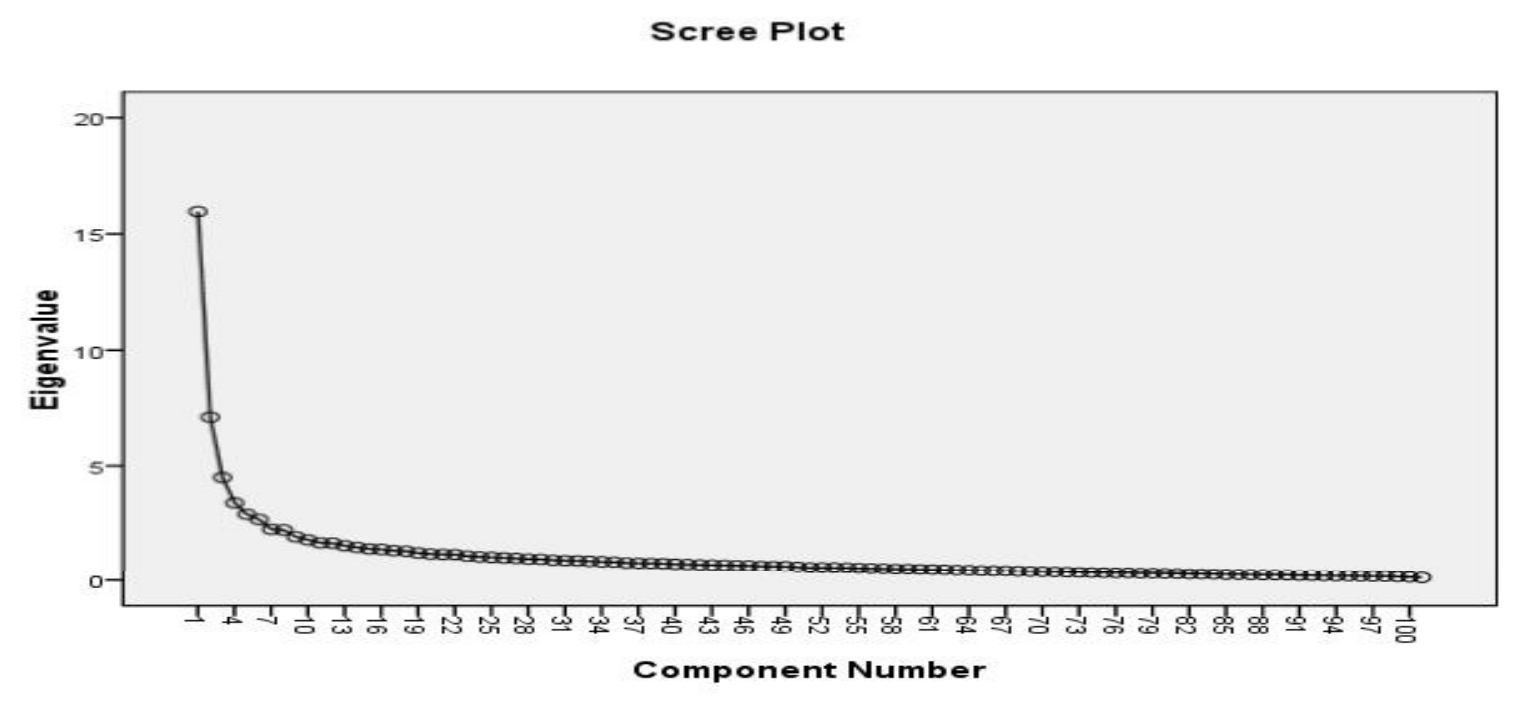

Figura 1. Scree Plot do CRQ.6.0.

De acordo com ela, o primeiro fator que emergiu ficou composto por 20 itens desenvolvidos originalmente para avaliar as Respostas do Eu; 14 migraram do componente Resposta do Outro e quatro de Desejo, totalizando 38 itens com cargas fatoriais entre $-0,68$ e 0,31 . Pode-se, portanto dizer, que o fator representa, prioritariamente, o componente RE. Quanto à polaridade, 13 itens de RE contemplam o polo positivo do construto, enquanto sete itens, o pólo negativo. Como exemplo de item positivo pode-se citar o item 28 (eu me sinto aceito pelo meu parceiro) e como exemplo de negativo, o 17 (eu me sinto arrasado sobre o relacionamento com o meu parceiro). Dentre os 14 itens que representam o componente RE, cinco também apresentaram carga fatorial $(>0,30)$ no segundo fator, constituindo-se, portanto em itens complexos (Pasquali, 1999). São eles: 18RE. Eu me dedico ao meu parceiro; 14RE. Eu sinto que meu parceiro é importante para mim; 2RE. Eu encorajo meu parceiro; 33RE. Eu fico ligado ao meu parceiro; 20RE. Eu compartilho meus sentimentos. Como todos eles tiveram maior carga no fator 1 e são teoricamente coerentes, sugere-se que sejam, por ora, mantidos como representantes de RE, na versão em português do CRQ 6.0, até que novas pesquisas possam corroborar ou não a adequação deles neste componente. Nessa medida, o componente $\mathrm{RE}$ da versão em português do CRQ fica representado por 20 itens, distribuídos em 13 itens positivos - 1RE, 2RE, 9RE, 12RE, 14RE, 18RE, 20RE, 24RE, 25RE,
27RE, 28RE, 31RE e 35RE - e sete negativos - 4RE, 7RE, 15RE, 17RO, 21RE, 23RE e 37RE. Quanto aos 14 itens de $\mathrm{RO}$ e quatro itens do componente $\mathrm{D}$, que apresentaram carga no primeiro fator, não devem ser aproveitados na composição da versão em português do CRQ 6.0.

O segundo componente ficou composto por 24 itens com cargas variando entre 0,38 e 0,69 . Nele, predominaram itens do componente Desejo $(n=19)$, dos quais apenas um também teve carga fatorial superior a 0,30 no fator 1: 39D. Eu gostaria de ajudar meu parceiro. Os demais itens migraram de outros componentes: três do componente Resposta do Eu e dois do componente Resposta do Outro. Adotou-se, para o fator 2, o mesmo critério de reter provisoriamente, também o item complexo, uma vez que teve maior carga fatorial ali e ele é teoricamente coerente. Quanto aos itens de RE e RO, devem ser descartados para a versão do CRQ em português. Nesta medida o componente Desejo fica representado por 19 itens: 28D; 22D; 34D; 32D; 37D; 30D; 29D; 26D; 13D; 20D; 19D; 23D; 11D; 39D; 01D; 18D; 15D; 16D; 12D.

O terceiro componente, cuja expectativa seria a Resposta do Outro, emergiu diferente do esperado. Com um total de 15 itens, reuniu seis itens originalmente desenvolvidos para medir as Resposta do $\mathrm{Eu}$, cinco de Desejo e quatro de Resposta do Outro. Suas cargas variaram entre 0,38 e 0,71 , todas positivas e não houve itens complexos. Procurando interpretar o 
fator, observa-se que ele reúne itens relacionados ao controle ou à dominação de um parceiro pelo outro. Seja como uma percepção de que o indivíduo tem controle sobre o parceiro, como, por exemplo, nos itens: 31RE. Eu domino meu parceiro, 30RE. Eu controlo meu parceiro, ou 06RE. Eu tenho poder sobre meu parceiro; seja como a percepção de que ele é controlado ou dominado pelo parceiro, como, por exemplo, os itens, 06RO. Meu parceiro me domina, 16RO. Meu parceiro me controla, 11RO. Meu parceiro tem poder sobre mim. Há ainda itens sobre o desejo de controlar ou ser controlado pelo outro, como nos itens, 40D. Eu desejo ter poder sobre meu parceiro, 10D. Eu desejo controlar meu parceiro, ou 38D. Eu desejo deixar meu parceiro tomar decisões por mim. Como as cargas dos itens são fortes nesse fator e o elemento de controle pode ser relevante num conflito interpessoal, sugere-se que sejam mantidos na versão em português do CRQ 6.0, como representantes da dimensão "Controle no Relacionamento- CR", entendida como a percepção ou o desejo de controle ou domínio de um dos parceiros envolvidos na relação. Nessa medida o terceiro fator fica composto pelos seguintes itens: 31RE; 30RE; 06RE; 40D; 10D; 06RO; 36RE; 16RE; 21D; 16RO; 11RO; 3RO; 35D; 13RE; 38D.

\section{DISCUSSÃO}

Esta pesquisa teve como objetivo principal estudar a estrutura fatorial do CRQ 6.0, um instrumento desenvolvido para avaliar o padrão de relacionamento conflituoso com o parceiro amoroso. Trata-se de iniciativa original, uma vez que os estudos anteriores, com esta versão do instrumento, forneceram medidas de consistência interna e de validade convergente e divergente (Barber et al., 1998; Massei \& Yoshida, 2009; Risso \& Yoshida, 2010; Silva \& Yoshida, 2009; Weinryb et al., 2000), e, em alguns deles, também a precisão de teste e reteste (Barber et al., 1998; Weinryb et al., 2000). Nessa medida, as comparações entre os resultados do presente estudo com aqueles só poderão ser feitas entre as avaliações dos escores médios e da consistência interna.

Conforme indicado, não foram encontradas diferenças entre os escores totais dos três componentes (D, RO e RE), quando a amostra foi dividida de acordo com o sexo, tipo de universidade (pública ou privada), ou os três estados da federação em que as amostras foram colhidas: PR, SP e SC. O equilíbrio nas médias em relação ao sexo era esperado, uma vez que não há razões teóricas para esperar níveis mais ou menos elevados de conflito no relacionamento amoroso em função desta variável, numa amostra de universitários. O mesmo pode-se dizer do tipo de universidade, ou dos três estados da federação, cujas populações têm características socioculturais semelhantes. Pode-se, no entanto conjecturar, se estas afirmações valeriam para amostras provenientes de outras unidades da federação, em que diferenças de ordem sociocultural tendem a afetar mais os padrões relacionais das pessoas em função do sexo. Esta é, portanto, uma questão em aberto que deverá ser respondida com amostras mais diversificadas em função dos estados brasileiros. De toda forma, é preciso lembrar, que também não foram encontradas diferenças significantes entre os escores médios dos demais estudos em que este cálculo foi realizado: com mulheres vítimas de violência e de maternidade (Silva \& Yoshida, 2009), de portadores de HIV/AIDS e acompanhantes (Massei \& Yoshida, 2009) e de portadores de Hepatite C crônica e acompanhantes (Risso \& Yoshida, 2010). Nessa medida, é também possível pensar que, apenas com base no escore total dos componentes do CRQ 6.0, não é possível discriminar diferentes estratos da população. Devendo-se, antes, analisar a configuração do padrão relacional mais característico, a partir da análise integrada, das respostas aos itens de cada componente. Esta, aliás, é uma alternativa mais condizente com a ideia de padrão conflituosos de relacionamento, conforme proposto por Luborsky (1984), para o CCRT.

O estudo da consistência interna dos itens dos três componentes apontou valores de alfas de Cronbach $(\mathrm{D}=0,86, \mathrm{RO}=0,82$ e $\mathrm{RE}=0,81$ ) muito semelhantes aos das pesquisas anteriores, em que os valores tenderam a superar 0,80 (Barber et al., 1998; Massei \& Yoshida, 2009; Risso \& Yoshida, 2010; Silva \&Yoshida, 2009; Weinryb et al., 2000). Isto é, indicando boa consistência entre os itens de cada componente e também da escala total, que no presente estudo foi 0,92 .

Quanto à validade de construto, os resultados do estudo obtidos por meio da análise fatorial apontaram para uma solução de três fatores, que atende parcialmente as expectativas teóricas. Os dois primeiros fatores medem, respectivamente, as Respostas do Eu e os Desejos, e corroboram parcialmente as versões: americana (Barber et al., 1998) e sueca (Weinryb et al., 2000) do instrumento. Quanto ao terceiro fator, mede 
o controle entre os parceiros no relacionamento amoroso e não as Respostas do Outro, conforma o esperado. É possível que a diferença se deva a questões de ordem cultural, devido aos valores mais tradicionais dos brasileiros, em que a submissão ao outro pode, eventualmente, ser entendida como uma "prova de amor"; "As pessoas ainda desejam um relacionamento que seja duradouro e entendem esta relação como um vínculo privilegiado entre os demais, ideias advindas do amor romântico... Diante de um mundo visto como cada vez mais ameaçador, os pós-moder-nos desejam alicerces, como uma relação amorosa" (Vieira \& Stengel, 2010, p. 158). Ainda que essas conjecturas careçam de evidências empíricas mais contundentes, vale lembrar que diferenças nos padrões relacionais amorosos de casais americanos e suecos em relação aos brasileiros, foram verificadas (Weinryb et al., 2000).

Com base nos resultados da presente pesquisa propõe-se que para avaliar o padrão de conflito amoroso de universitários da região sul e sudeste do país, se utilize a versão do CRQ 6.0 com os componentes que emergiram da análise fatorial. Isto é, uma versão composta pelos 20 itens de RE, 19 itens de D e 15 itens para o componente Controle no Relacionamento (CR), num total de 54 itens. Quanto ao componente RO, estaria por ora parcialmente contemplado pelos itens de CR que aferem aspectos das respostas do outro. No entanto, propõe-se o prosseguimento das investigações com o desenvolvimento de novos itens que venham complementar a avaliação do padrão relacional conflituoso e novas análises da estrutura fatorial do instrumento.

Sugere-se ainda que se busque empregar amostras mais variadas, integradas por estudantes de outras regiões do país, além de pessoas de outros estratos da população, tais como casais atendidos em varas da família que se encontram em situação de litígio e separação; pessoas portadoras de doenças físicas cujos sintomas podem afetar ou comprometer o relacionamento amoroso; pessoas que buscam psicoterapias devido a problemas de relacionamento conjugal, entre outras. O acúmulo de evidências das propriedades psicométricas do CRQ deve, portanto prosseguir, para que seus limites e aplicabilidade venham a ser melhor conhecidos.

Finalmente, cabe uma palavra sobre a forma de avaliação e interpretação desse instrumento. Nenhum estudo publicado, até o momento, informa sobre os procedimentos de avaliação do CRQ. Os procedimen- tos de avaliação são importantes para que o usuário siga normas padronizadas. A padronização para aplicação de testes psicológicos, inventários, escalas são fundamentais para que não se incorra em erros de interpretação "padronização implica em uniformidade de procedimento na aplicação e pontuação dos testes" (Anastasi \& Urbina, 2000, p. 20). Assim, compreende-se que sua aplicação como instrumento de avaliação fica comprometida até que um manual seja desenvolvido estabelecendo padrões de aplicação. Considerando que ele deriva do CCRT, entende-se que cada componente deva ser primeiramente avaliado de forma independente e, só então, deve-se buscar compreendê-lo em relação aos demais, numa tentativa de se traçar o padrão de relacionamento característico daquela pessoa. Além disso, é muito provável que em situações clínicas outras fontes de avaliação devam ser levadas em conta, para a definição mais precisa da natureza e da amplitude do conflito amoroso sobre o funcionamento geral do indivíduo.

\section{REFERÊNCIAS}

Anastasi, A., \& Urbina, S. (2000). Testagem psicológica. Porto Alegre: Artmed.

Barber, J. P., Foltz, C., \& Weinryb, R. M. (1998). The Central Relationship Questionnaire: Initial Report. Journal of Counseling Psychology, 45, 131-142.

Crits-Christoph, P., Barber, J. P. Grenyer, B. F. S., \& Diguer, L. (2010). Lester Luborsky: A trailblazer in empirical research on psychoanalytic therapy. In L. G. Castonguay, J. C. Muran, L. Angus, J.A. Hayes, N. Ladany, \& T. Anderson (Eds.), Bringing psychotherapy research to life: Understanding change through the work of leading clinical researchers (pp.39- 49). Washington, DC: American Psychological Association.

Laloni, D. T. (2001). Escala de Avaliação de Sintomas-90-R-SCL90-R: Adaptação, precisão e validade (Tese de Doutorado). Pontifícia Universidade Católica de Campinas. Retirado em 22 de fevereiro de 2011, de www.bibliotecadigital.puccampinas. edu.br/tde_busca/arquivo.php?codArquivo=294

Lhullier, A. C. (1998). O Tema Central de Conflito de Relacionamento. In A. C. Lhullier (Ed.), Novos modelos de investigação em psicoterapia (pp. 55-98). Pelotas, RS: Educart.

Luborsky, L. (1984). Principles of psychoanalytic therapy: A manual for supportive-expressive treatment. New York, NY: Basic Books.

Luborsky, L., \& Crits-Christoph, P. (Eds). (1998) Understanding transference: The core conflcitual relationship theme method. Washington DC: American Psychological Association.

Massei, A. C., \& Yoshida, E. M. P. (2009). Avaliação das propriedades psicométricas do Questionário de Relacionamento Central 6.0 (CRQ 6.0) em portadores de HIV/Aids. Psicologia: Teoria e Prática, 11, 83-96. 
McCarthy, K. S., Gibbons, M. B. C. P., \& Barber, J. P. (2008). The relation of rigidity across relationships with symptoms and functioning: An investigation with the revised Central Relationship Questionnaire. Journal of Counseling Psychology, 55, 346-358.

Pasquali, L. (Ed.) (1999). Instrumentos psicológicos: Manual prático de elaboração. Brasília: LabPAM/IBAPP.

Pasquali, L. (2001). Princípios de elaboração de escalas psicológicas. In C. Gorenstein, L. H. S. G. Andrade \& A.W. Zuardi (Eds.), Escalas de avaliação clínica em psiquiatria psicofarmacológica (pp. 15-22). São Paulo: Leitura Médica.

Risso, G., \& Yoshida, E. M. P. (2010). Validade e precisão do Questionário de Relacionamento Central 6.0 (CRQ 6.0) para adultos com hepatite C crônica. Paidéia, 20, 219-227.

Rocha, G., M., A. (2007). Tradução e adaptação cultural do Central Relationship Questionnaire. Projeto de pesquisa (manuscrito) com registro no SINEP sob n. 0015.0.272.000.07. São Paulo.
Silva, F. R. C. S., \& Yoshida, E. M. P. (2009). Questionário de Relacionamento Central - CRQ 6.0: Estudo exploratório de validade com mulheres vítimas de violência. Avaliação Psicológica, 8, 405-414.

Vieira, E. D., \& Stengel, M. (2010) Os nós do individualismo e da conjugalidade na pós-modernidade. Aletheia, 32, 147-160.

Weinryb, M. R., Barber, J. P., Foltz, C., Göransson, S. G. M., \& Gustavsson, J. P. (2000). The Central Relationship Questionnaire (CRQ): Psychometric properties in a Swedish sample and cross-cultural studies. Journal of Psychoterapy Practice and Research, 9, 201-212. 